\title{
Une lecture des Adages d'Érasme, entre bibliographie rêvée et bibliothèque idéale
}

\author{
Isabelle Diu \\ École nationale des Chartes (Paris)
}

Les Adages d'Érasme ont représenté l'un des plus grands succès de librairie de la Renaissance ${ }^{1}$ : cet ouvrage, en chantier permanent durant la vie de l'humaniste 2 , a fait l'objet d'un

1 L'édition de référence des Adages est aujourd'hui celle des œuvres complètes d'Érasme, en cours de publication. Les Adages comprennent six volumes : Opera omnia Desiderii Erasmi Roterodami, Amsterdam, 1969-, II, 16. Cette édition sera désignée par le sigle A.S.D. À noter également que les Adages sont disponibles en traduction anglaise intégrale dans les Collected Works publiés aux Presses universitaires de Toronto. Mentionnons également la publication, à l'automne 2011, de l'édition bilingue intégrale des Adages aux Belles Lettres sous la direction de Jean-Christophe Saladin.

2 Les lettres où l'humaniste avoue remettre l'ouvrage sans cesse sur le métier sont légion, notamment les préfaces à ses différentes éditions. Voir P. S. Allen, 
nombre considérable d'éditions, du vivant même de son auteur; il a rencontré un succès prodigieux auprès d'un large public comme dans les cercles érudits ${ }^{3}$. Les éloges incessants dont les milieux humanistes ont couvert l'ouvrage ont contribué à bâtir la renommée d'Érasme et à asseoir définitivement sa réputation comme homme de lettres ${ }^{4}$. Les raisons en sont faciles à comprendre : d'un accès aisé, presque ludique, composé comme en passant ${ }^{5}$, l'ouvrage semble véhiculer une sorte de sagesse populaire ; pourtant, il s'appuie sur les autorités les plus prestigieuses, révèle de hautes qualités rhétoriques et s'avère ancré dans la réalité la plus contemporaine. Bien loin de n'être qu'une simple compilation à usage éthique, l'œuvre ressortit à la catégorie des essais rhétoriques et esthétiques. D'un même mouvement, il met à portée du grand nombre, avec un réel talent pédagogique, les trésors de la sagesse antique, sous une forme nouvelle et proprement humaniste : en restituant la lettre même des textes. Cette sorte d'anthologie de la littérature antique est donc aussi une forme de manifeste de l'humanisme lettré.

Les analyses récentes des Adages se sont attachées à mettre en valeur son caractère linguistique et rhétorique : pour Jacques Chomarat, l'œuvre se veut un «manifeste des droits de

1906-1958, 12 vol. Les renvois à cette édition seront indiqués comme suit : Allen, suivi du tome et du numéro de la lettre. Les préfaces aux Adages sont les suivantes : I, 126 ; I, 211 ; I, 269 ; IV, 1204 ; VI, 1659 ; VII, 2022 ; X, 2773.

3 T. J. Arnold, 1897 : sous le titre Adagiorum Collectanea, on dénombre trentecinq éditions avant 1536 ; les éditions des Chiliades sont au nombre de seize. Un grand nombre d'épitomè ont également contribué à la diffusion de l'œuvre : quinze éditions abrégées ont paru du vivant d'Érasme.

4 Pour une étude d'ensemble de l'œuvre, on se reportera à Phillips, 1964.

5 Voir la préface à la première édition en 1500 (Allen, I, 126), où Erasme se compare au promeneur qui cueillerait des fleurettes dans un jardin pour en tresser une guirlande. 
la grammaire » qui se dégage d'une silva, forêt broussailleuse, d'un supellex ou matériau constitué de toutes les expressions utiles à l'écrivain et frappantes pour le curieux (p. 761-782) ; pour Claudie Balavoine, l'ouvrage se situe «sur le plan du langage et du savoir, non de la conduite morale » (p. 9-23) ; il cristallise finalement autour de l'adage toute une conception de la culture. Cependant, le texte même des Adages, son contenu comme sa structure, ainsi que la forme matérielle qu'affectent les diverses éditions du texte imprimé, invitent, en raison de leur complexité, à une lecture polysémique. On peut notamment se demander si cet ouvrage multiforme ne serait pas aussi à lire comme une tentative bibliographique, emblématique de l'humanisme, à laquelle Érasme lui-même et ses imprimeurs nous inviteraient, rassemblant les autorités idéales en un panthéon des auteurs classiques et de leurs grands textes. Nous nous aventurerons donc à cette lecture, en considérant tout d'abord les Adages comme le lieu de construction d'un canon bibliographique, puis en confrontant ce modèle idéal avec les lectures d'Érasme, repérables par le biais de bibliothèques de prédilection - la sienne propre, mais aussi celles d'autres cercles humanistes - et à travers l'énoncé de ses choix littéraires.

\section{L'évolution des enjeux au fil des éditions}

On ne saurait séparer l'étude du texte des Adages de celle des diverses éditions qui les ont fait connaître: les enjeux de l'ouvrage rejoignent en effet les choix éditoriaux et le contenu de l'œuvre évolue en fonction de sa mise en texte. 
Les Adages ne sont pas seulement le premier recueil de proverbes latins, même si Érasme le présente comme tel ${ }^{6}$; ils s'affirment surtout comme une première tentative pour ouvrir, par le biais des proverbes, des perspectives sur le monde antique. L'œuvre affecte une forme assez codifiée : les adages choisis sont tirés pour l'essentiel des grands auteurs classiques de l'Antiquité, auxquels s'ajoutent quelques citations bibliques et patristiques ou, plus rarement, des textes d'auteurs contemporains. Les adages sont d'abord reproduits sous leur forme latine et, le cas échéant, leur forme grecque; ils sont suivis d'autant de références qu'Érasme avait pu en recueillir chez les auteurs qu'il avait à sa portée. Les citations se présentent souvent accompagnées d'un renvoi précis à l'œuvre, $\mathrm{au}$ chapitre, au vers ou au verset. L'importance, au moins théorique, attachée à la précision des références est attestée par la préface de la nouvelle édition parue en 1526 dans l'atelier de Froben à Bâle (Allen, 1906, VI, 1659). Cependant, en dépit de la revendication d'un authentique travail d'écriture (negocium) aboutissant à une œuvre (opus), suivant une

\footnotetext{
6 Voir les différentes préfaces aux éditions des Adages. L'édition princeps des Adagiorum collectanea signale, dès 1500, la nouveauté de l'entreprise, du moins chez les Latins : "Porro apud Latinos nemo quidem ante nos, quod sciam, huiusmodi negocium tentavit »; " Chez les Latins, personne avant moi, que je sache, n'a tenté une entreprise de ce genre » (Allen, I, 126, l. 95-96) ; dans la préface à la troisième édition, celle des Chiliades parue en $1515 \mathrm{chez}$ Froben, Érasme réitère l'affirmation (Allen, I, 269, l. 58) ; enfin, la dédicace de la dernière édition à laquelle il met la main de son vivant, en 1533, est presque entièrement consacrée à la défense de sa primauté comme auteur de recueils d'adages : s'il reconnaît avoir lu l'ouvrage de l'Italien Polydore Virgile, initialement paru sous le titre de Proverbiorum libellus en 1498, puis réédité en 1500 au même moment que les Adagiorum collectanea avec un intitulé comparable - Adagiorum liber - Érasme revendique néanmoins la première place. Négligeant l'édition de 1498, peut-être en toute bonne foi, il prétend avoir l'antériorité de la publication de ce type d'ouvrage et clame sa supériorité quant à la qualité et à l'ampleur de l'œuvre (Allen, X, 2773).
} 
méthode précise (ratio) ${ }^{7}$, Érasme n'impose aucun ordre à ce qui se présente comme une libre succession de citations ; l'ouvrage se veut miscellané, à la façon des Nuits attiques d'Aulu-Gelle ou des Saturnales de Macrobe, qui figurent d'ailleurs au nombre de ses sources.

Cette mosaïque de références et de commentaires n'offre, dans le corps du texte, aucune explication didactique sur le genre de l'œuvre ou sur la nature de l'adage. Pour trouver la définition du proverbe selon Érasme, le lecteur doit se reporter à deux textes liminaires : la préface de la première édition des Collectanea, en 1500, dédiée à lord William Mountjoy (Allen, I, 126), et l'introduction, intitulée Quid sit paroemia, souvent désignée sous le titre de Prolegomena, qui précède le texte des Adages à partir de l'édition aldine de 1508 (ASD, 1969, II, 1, p. 45-82). Deux caractéristiques distinguent, selon Érasme, les expressions qu'il recueille et auxquelles il accorde valeur proverbiale : leur notoriété et leur originalité ${ }^{8}$. Ainsi Érasme peut-il orienter son ouvrage comme il l'entend, fonder ses choix sur sa conception du langage et de la rhétorique et ne se cantonner ni à une nomenclature de type lexicographique ni à un recueil de pensées gnomiques. C'est dire toute l'importance que revêtent ces discours théoriques, lors même qu'ils sont apparemment relégués en dehors du texte, dans le discours paratextuel. Outre la définition des adages, le but de l'ouvrage s'y voit également précisé : s'il s'agit de transmettre au public

\footnotetext{
7 Allen, I, 126, l. 96 et l. 199 : «Reliquum esse puto ut in opere nouo quid secuti simus rationem reddamus »; «Il me reste, je pense, à rendre compte de la méthode que j'ai suivie dans un ouvrage d'un genre tout nouveau ». 8 « Paroemia est celebre dictum, scita quapiam novitate insigne », Quid sit paroemia, ASD, II, 1 ; p. 46, l. 44-45. La traduction qu'en donne Claudie Balavoine (p. 13) est d'une parfaite précision : «L'adage est une expression connue qui se distingue par quelque originalité spirituellement savante ».
} 
moderne la sagesse du passé, c'est seulement au travers de grands textes, que distingue la grâce de leur eloquentia. En parallèle, il convient d'inculquer au lecteur le sens de l'histoire, de l'aider à acquérir la notion de la perspective, en présentant les Anciens dans l'individualité distinctive de leur temps, tout en postulant l'invariance de la nature humaine. Ce point de vue, moderne par rapport au Moyen Âge, qui ne considère les événements passés que dans son propre univers mental, est emblématique d'une culture proprement humaniste, qui tisse constamment les fils du texte des Adages. La triple visée de l'œuvre - mise en lumière des classiques, tant dans leur contenu textuel que dans leur forme rhétorique ; affirmation de la continuité de la pensée; actualité des textes anciens rejoint les constantes préoccupations philologiques, rhétoriques mais aussi politiques de l'humaniste.

Ces éléments - texte, paratexte, mais aussi mise en texte et mise en images - , qui se conjugueront pour donner leur physionomie définitive aux Adages d'Érasme à la fin du XVI siècle, ne se présentent pourtant pas d'emblée dans le petit ouvrage publié en 1500 à Paris sous le titre d'Adagiorum collectanea. Ils surgissent à mesure que se succèdent les différentes éditions des Adages, la matérialité du texte contribuant à façonner peu à peu le contenu de l'œuvre tandis qu'en retour, le discours se coule dans une forme toujours plus adaptée à sa visée.

L'édition princeps est celle des Adagiorum collectanea, publiée à Paris en juillet 1500, chez l'imprimeur Iohann Philippi, lors du séjour d'Érasme dans la capitale (BNF Res Z 447). Petit in-quarto de quelques dizaines de feuillets, s'ouvrant sur une epistola nuncupatoria de Fausto Andrelini, poète italien 
installé à Paris, il comporte également, en guise de paratexte, l'importante préface d'Érasme dédiée à son ancien élève, devenu son protecteur, l'humaniste anglais lord Mountjoy; cette lettre, comme nous l'avons rappelé, inscrit d'emblée l'œuvre dans une ambitieuse perspective, s'appuyant sur les grands principes de la culture humaniste pour proposer une relecture des auteurs antiques. Si ce dessein ne sera finalement pleinement réalisé que dans les éditions ultérieures, il existe pourtant déjà à l'état latent dans l'édition originale. Cette première publication des Adages correspond à une nouvelle ère dans le commerce du livre parisien; elle débute avec la diffusion massive d'œuvres humanistes, imprimées à l'aide de caractères romains et non plus gothiques, par des imprimeurs ambitieux comme Josse Bade, les Estienne ou Gilles de Gourmont (Martin, 2000, p. 180 sq). En 1500, en effet, il est encore hasardeux, pour l'auteur comme pour son imprimeur, de mettre sur le marché un volume concernant uniquement ou presque les classiques, imprimé de surcroît avec des caractères peu familiers aux yeux du public. Fontes d'imprimerie et contenu sont, là aussi, intimement liés; le format se révèle également original, empruntant la forme d'un ouvrage de poche, aisément maniable, qui prétend mettre la sagesse antique, comme l'éloquence des classiques, à la portée de tous. L'ouvrage, de fait, permet à un public élargi, qui ne se borne pas aux érudits les plus privilégiés, d'avoir accès aux autorités classiques et surtout à leurs textes mêmes, à ce "jardin d'auteurs variés », pour reprendre les termes de la préface d'Érasme, où il prétend avoir cueilli son florilège. Un grand nombre d'auteurs antiques, qui viennent seulement d'être publiés en Italie, ou ne circulent encore que sous forme manuscrite dans des cercles restreints, reçoivent un éclairage 
particulier grâce à l'œuvre d'Érasme, lors même qu'ils restent encore inaccessibles à la majorité des lecteurs.

Quelques années plus tard, une nouvelle édition voit le jour à Venise, chez Alde Manuce, où Érasme s'est rendu à la fin de l'année 1507, emportant ses traductions d'Euripide qu'il espère faire publier par le grand imprimeur vénitien. La nouvelle version des Adages débute en 1508 et est menée à bien fébrilement en l'espace de quelques mois. Pourtant, en ce court laps de temps, l'ouvrage s'est totalement métamorphosé : il est devenu monumental et a adopté sa forme définitive, même s'il se verra encore considérablement augmenté au cours des éditions qui suivront. En premier lieu, le livre change de titre, ce qui dénote un changement non seulement quantitatif, mais aussi qualitatif: il s'intitule désormais Adagiorum chiliades. L'accent est mis sur l'importance du contenu, qui rassemble plusieurs milliers de proverbes (chiliades), classés par dizaines, centaines et milliers. En outre, cette édition propose une nouvelle vision des classiques et postule l'existence des Belles Lettres dans leur continuité : le but de l'ouvrage n'est plus seulement d'expliciter tel ou tel proverbe, mais de donner son pedigree, de montrer qu'il vit d'un auteur à l'autre. Le caractère d'anthologie de l'ouvrage date de cette édition des Chiliades, qui présente de larges passages des grands textes antiques, comme l'Iliade par exemple, dans leur intégrité et non sous forme de résumé. Le but de cette édition est donc de propager ce qui représente aux yeux d'Érasme la "bonne littérature», de mettre en lumière les autorités auxquelles puisse se référer la République des lettres, en exerçant toutefois son acribie et en admettant qu'il n'est pas d'autorité infaillible. 
Les éditions ultérieures suivent une expansion continue, de 1515 à 1533 ; seule la dernière, préparée par Érasme luimême, en 1533, verra se réduire non tant la quantité des adages que le nombre des auteurs cités. La troisième grande édition à voir le jour est celle de Bâle, en 1515, chez Froben, qui publie à nouveau l'ouvrage sous le titre de Chiliades; c'est le premier texte donné par Érasme aux presses de l'imprimeur bâlois ${ }^{9}$. Le contenu de l'œuvre s'enrichit de l'ajout d'un certain nombre de proverbes, et notamment du fameux Dulce bellum inexpertis, qui connaîtra une fortune singulière et sera à plusieurs reprises publié seul, chez divers imprimeurs. Les Adages de 1515 placent en effet Érasme au centre des discussions sur les questions sociales et politiques, de même que le Nouveau Testament, en 1516, lui conférera une position inégalée dans le renouveau de l'étude textuelle de l'Écriture. Surtout, sur le plan formel, cette édition se distingue par un paratexte et une mise en page exceptionnelle, sur lesquels nous reviendrons ${ }^{10}$.

Plusieurs autres éditions bâloises paraissent entre 1517 et 1528, proposant ajouts et corrections, jusqu'à l'Adagiorum opus de 1533. Cette dernière édition est rédigée au milieu des attaques de la maladie et des atteintes de la vieillesse, alors qu'Érasme a dû se réfugier à Fribourg en raison des troubles incessants que suscite le triomphe du parti réformé à Bâle. L'humaniste semble accorder une attention plus particulière à certains auteurs, sans doute en raison de la pénurie de livres dans sa retraite fribourgeoise, mais peut-être aussi pour façonner différemment l'ouvrage, se focalisant sur certains

\footnotetext{
9 Iohann Froben avait déjà publié deux ans auparavant une copie de l'édition vénitienne de 1508. L'édition de 1515 est bien une nouvelle édition, augmentée et procurée avec l'aveu d'Érasme.

10 Nous avons pu consulter la réédition de 1518 : BNF Res g Z 37.
} 
grands textes et délaissant le butinage dans un vaste champ. Enfin, une ultime édition verra le jour du vivant d'Érasme, en 1536. Dans l'édition posthume de ses Opera omnia en 1540, les Adages occupent l'intégralité du second tome d'une édition qui en comprend neuf, belle illustration de l'importance prise par ce texte au sein de l'œuvre de l'humaniste (BNF Res Z 323 tome 2).

À l'évolution du contenu des Adages répond très exactement celle de leur mise en texte. La présentation formelle des proverbes atteint sa forme définitive entre la première et la seconde édition. En 1500, le texte s'ouvre sur une référence à un auteur classique, que suit l'explication de l'adage, puis le recours à d'autres références. Cette disposition sera abandonnée dans les éditions ultérieures, qui débuteront toujours par la citation du proverbe lui-même, suivi d'une courte explication, puis de l'énumération d'une série d'autorités. Mais même si le discours se veut fluide, si le choix d'un arrangement souple des références incite à une lecture continue, l'œuvre est également pédagogique: la nécessité d'appareiller l'ouvrage à l'aide d'instruments de lecture se fait rapidement sentir et se voit explicitement formulée. Dès 1508, Érasme, pour l'atelier d'Alde, procède à l'adjonction de deux index, placés avant même le début du texte, l'un alphabétique, l'autre thématique (iuxta locos), qui permet une utilisation rhétorique du matériau qu'il rassemble ; ces index seront repris dans toutes les copies ou les éditions ultérieures. Leur nécessité est précisée en quelques lignes introductives. Érasme soulignera à nouveau leur importance dans l'une de ses 
préfaces ${ }^{11}$. Une démarche semblable préside à l'établissement des sources des Adages: dans le cours de l'ouvrage, Érasme ne donne jamais les références exactes de ses citations. S'il précise parfois l'œuvre, le chapitre ou le vers qu'il cite, le plus souvent, il mentionne seulement le nom de l'auteur. Il y remédie ailleurs, dans les écrits paratextuels qui accompagnent, nous l'avons dit, sa compilation. En 1500, dans sa lettre de dédicace à lord Mountjoy, il cite, sans ordre défini, bon nombre de ses auteurs de prédilection, dont il relève le goût pour l'adage. De même, dans les deux longs essais ajoutés en 1508, Herculei labores et Festina lente, qui présentent, outre un long développement explicatif sur chacune de ces deux expressions, un intéressant métadiscours sur la composition de l'œuvre, il livre certaines indications sur ses sources: dans le premier, une liste des auteurs utilisés pour collecter ses proverbes, dans l'autre une nomenclature des manuscrits qu'il a pu consulter à Venise. Enfin, à partir de l'édition procurée par Froben en 1515, un véritable catalogue des auteurs précède le texte, même si cette liste ne rassemble qu'une partie des autorités qui seront de fait utilisées; elle s'accompagne d'une "mise en image» de ce catalogue dans le frontispice, sur laquelle nous allons revenir.

\section{Le lieu de construction d'un canon bibliographique}

Ce sont précisément ces autorités et la manière dont elles sont mises en lumière par Érasme et ses imprimeurs en une sorte de bibliographie idéale qu'il nous faut maintenant examiner. Érasme s'assigne en effet la tâche, même si elle n'est présentée

11 Allen, VI, 1659 ; l. 50-54 ; il y appelle les imprimeurs à veiller à la correction de ces index et à l'exactitude des références qu'ils offrent. 
qu'en creux, d'établir un véritable catalogue (catalogus ou recensio) des auteurs d'adages.

Dès 1500, dans la préface de la première édition dédiée à lord Mountjoy, il mentionne les nombreux auteurs auxquels il a fait appel: classiques grecs ou latins, comme Platon, les dramaturges Plaute et Térence, Varron, les poètes Catulle, Horace, Perse, puis Martial et Ausone. Viennent ensuite Pline l'ancien, les rédacteurs de collections de proverbes grecs, AuluGelle et Macrobe, puis deux auteurs sacrés : Jérôme et Basile. Pour finir, il cite quelques modernes : Ermolao Barbaro, Pic de la Mirandole et Ange Politien. Et il termine son énumération sur ces mots : "pour ne pas prolonger ce catalogue au-delà des limites, concluons... $»^{12}$ (Allen, I, 126, l. 144-145). Le recensement n'est pas seulement rhétorique, il a aussi valeur instrumentale : il s'agit bien d'un catalogus.

En 1515, dans l'édition procurée par Froben, c'est sur un nouveau catalogus que s'ouvre doublement l'ouvrage (BNF : Rés. g Z 37) : le premier écrit paratextuel, qui se présente aux yeux du lecteur, juste après la page de titre et avant les deux index, est une liste, imprimée sur trois colonnes, d'auteurs grecs et latins, essentiellement antiques, arrangés selon un ordre précis. Vient d'abord la littérature grecque, divisée selon les catégories suivantes : poètes, historiens, orateurs, philosophes, théologiens et grammatici mixti où voisinent compilateurs et grammairiens. Puis les Latins, classés suivant les mêmes divisions mais dans un ordre quelque peu différent: poètes et historiens apparaissent toujours en tête, mais ils sont suivis des grammairiens, puis des orateurs; ensuite paraissent les mixti, comme Pline l'ancien, Aulu-Gelle, Macrobe, et enfin les «philosophes », catégorie qui

\footnotetext{
12 « verum ne longius catalogum proferam ».
} 
comprend les Pandectes et Columelle, puis deux modernes: Vitruve et Végèce. Cette liste de cent quatorze auteurs (soixantequatorze Grecs et quarante Latins) est précédée d'un avertissement: Autorum catalogus non omnium nam id fuerat bibliothecae indicem scribere, sed praecipuorum, e quorum monumentis collectae chiliades sunt Adagiorum. Cette indication établit une distinction majeure entre catalogue d'auteurs choisis (Autorum catalogus non omnium..., sed praecipuorum), c'est-àdire bibliographie sélective, d'une part, et catalogue de bibliothèque (bibliothecae index), qui supposerait une exhaustivité des références. Mais là encore, la liste, quoiqu'elle existe, bien qu'elle ait conquis son autonomie par rapport à la préface et soit de surcroît organisée clairement en rubriques, voit son importance aussitôt réduite : elle ne se prétend pas complète et il n'entre nullement dans les intentions de l'auteur ni de l'imprimeur de rédiger un véritable catalogue.

Pourtant, l'importance d'une nomenclature des auteurs est réaffirmée à deux reprises dans cette édition : la page de titre s'organise également autour d'un catalogue, restreint cette fois, comme pour bien marquer toute l'importance des autorités sous l'égide desquelles l'œuvre se place. Le titre, sous forme d'une adresse au lecteur (Ioannes Frobenius studiosis omnibus s.d. Accipito candide lector Erasmi Roterodami proverbiorum chiliadas...), est imprimé dans un cartouche au centre de la page, composée d'une gravure où des auteurs en buste dialoguent entre eux, arborant livres et instruments de musique, dans des galeries suggérant une sorte de cloître ou de demeure idéale, close sur un jardin arboré et ornée de fontaines. Le bandeau supérieur met en présence les deux grands poètes du panthéon grec, Homère et Hésiode, de part et d'autre de la figure emblématique de Salomon. Puis viennent 
par couple Aristide et Démosthène, Platon et Aristote, Euripide et Aristophane, Plutarque et Lucien, Théocrite et Pindare, Cicéron et Quintilien, Virgile et Horace, enfin Pline et Aulu-Gelle, Tite-Live et Salluste. On trouve là, sous une forme figurée, la plupart des grandes divisions du catalogus qui va suivre, poètes, orateurs, philosophes et auteurs de mélanges littéraires qui, dans cette architecture inaugurant le volume, figurent comme les piliers qui soutiennent la République des lettres.

Si l'on examine maintenant la liste des auteurs réellement utilisés par Érasme pour ses diverses éditions des Adages, on constate que la recension des auteurs les plus fréquemment cités recouvre presque exactement la liste initiale des grandes autorités revendiquées par Érasme dès 1500. Margaret Mann Phillips, dans l'ouvrage qu'elle publie sur les Adages d'Érasme en 1964, en se fondant d'ailleurs sur une étude menée par Théodor Appelt, restée inédite et intitulée Contents and sources of the Adages of Erasmus, recense, dans les tables imprimées à la fin de son ouvrage, trois cent soixante-trois auteurs cités par Érasme dans les diverses éditions des Adages, parmi lesquels nous retiendrons ceux dont il fait le plus fréquemment usage ${ }^{13}$. On laissera de côté les auteurs des grandes collections de proverbes grecs, que l'on peut considérer comme des sources trop spécifiques pour entrer dans le cadre de notre étude, même si elles font l'objet d'emprunts massifs : ce sont celles des grammairiens antiques, Diogénien (pour cent soixante-huit citations) ou Zénodote (pour deux cent trente), celle du rhéteur Athénée (trois cent cinquante-six occurrences) ou les compilations plus récentes de Suidas au $\mathrm{X}^{\mathrm{e}}$ siècle (trois cent

13 Phillips, 1964, p. 383-403. L'index nominum publié dans le volume I, 6 de l'édition A.S.D. est établi sur des critères trop larges pour être utiles ici. 
quatre-vingt-douze citations) ou encore d'Apostolius de Byzance, protégé du cardinal Bessarion (six citations seulement). Parmi les auteurs qui nous intéressent, viennent d'abord les poètes, grecs mais surtout latins, ces derniers offrant une plus grande diversité de sources. Homère fournit un nombre impressionnant de citations: six cent soixante-six; suivent Pindare (cent soixante-seize mentions), Théocrite (cent cinquante), Hésiode (quatre-vingt-huit) et Théognis (quarantesept). Parmi les Latins, les lyriques autant que les poètes épigrammatiques sont à l'honneur: Horace talonne presque Homère avec quatre cent soixante-quinze emprunts ; Virgile est cité deux cent vingt-neuf fois, Ovide cent vingt-cinq, Perse quatre-vingt-dix-neuf, Martial quatre-vingt-douze, Catulle trente seulement, Properce treize et Tibulle à peine six fois. Les auteurs dramatiques, comiques comme tragiques, fournissent aussi leur lot de proverbes: le lauréat est, sans surprise, Aristophane, qui livre cinq cent quatre-vingt-seize citations ; Euripide vient en seconde position avec deux cents citations, puis Sophocle (cent quinze), Ménandre (quarante-trois), enfin Eschyle, dans une moindre mesure (trente-six). Parmi les Latins, la palme revient à Plaute avec quatre cent soixantequinze citations - autant qu'Horace - , puis Térence avec deux cent cinquante-sept. Chez les philosophes, l'intérêt est également partagé entre Platon (quatre cent soixante-quinze citations) et Aristote (trois cent quatre). C'est dans cette catégorie qu'Érasme range également son auteur de prédilection, Plutarque, qui sans parvenir à égaler Homère, lui fournit néanmoins six cent dix-huit extraits. Il rejoint, parmi les moralistes, Lucien, qui contribue également grandement à la moisson de proverbes (trois cent trente-cinq citations). La liste des historiens rassemble quelques Grecs, Hérodote avec 
cinquante-trois mentions, Pausanias (vingt-neuf), Xénophon (treize), Thucydide, quoique fort peu (huit) ; les plus présents sont surtout les auteurs latins: Juvénal (cent vingt-sept citations), Tite-Live (soixante-seize), Suétone (trente-sept), Tacite (onze) et Salluste (dix). Parmi les orateurs, nous trouvons, sans surprise, Cicéron à la première place avec huit cent quatre-vingt-douze emprunts, Sénèque (cent vingt-six), Quintilien (quatre-vingt-sept), Pline le Jeune (trente-neuf), puis les Grecs, Démosthène (quarante mentions) et Isocrate (huit citations seulement). Érasme fait également appel à des autorités dans leur domaine, comme Strabon pour la géographie (soixante et onze citations), Galien pour la médecine (trente-cinq citations), de préférence à Hippocrate (deux citations) et Columelle pour l'agriculture (vingt-quatre). Enfin quelques auteurs passés maîtres dans l'art des miscellanées lui sont d'un grand secours : Pline l'Ancien, au premier chef, avec deux cent quatre-vingt-sept citations, dépasse Euripide ; AuluGelle le suit avec cent douze emprunts, puis Macrobe (quarante-trois citations). Les textes des Pères de l'Église sont très rarement cités, sauf Jérôme, qui apporte sa contribution cent vingt et une fois; on ne trouve en revanche que vingt-trois citations de Basile, vingt et une de Chrysostome, dix-sept de Grégoire de Nazianze, huit d'Origène et trois d'Ambroise, le grand absent étant Augustin.

\section{Bibliographie, bibliothèque d'Érasme et bibliothèque} humaniste

Reprenant la distinction, suggérée par Érasme, entre constitution d'une bibliographie et établissement d'un 
catalogue, nous voudrions maintenant nous interroger sur les rapports de ce canon bibliographique, esquissé dans les Adages, avec la réalité des bibliothèques humanistes. Si la bibliographie relève d'un modèle idéal de bibliothèque, s'appuyant sur un modèle bibliographique reconnu par tous, quelles sont les relations qu'entretient le panthéon des auteurs réunis dans cette bibliographie avec la bibliothèque privée d'Érasme, les bibliothèques des cercles érudits humanistes, voire sa collection idéale personnelle?

Nous connaissons la composition de la bibliothèque d'Érasme grâce à deux inventaires manuscrits, conservés à la bibliothèque universitaire de Bâle ${ }^{14}$. Le plus important consiste en une liste d'ouvrages d'auteurs antiques et contemporains, intitulée «Versandliste » ou liste d'envoi, probablement établie par l'un des secrétaires de Boniface Amerbach, l'ami et exécuteur testamentaire d'Érasme, avant l'envoi de la bibliothèque érasmienne à l'humaniste polonais Jean Laski, qui

14 Sur la bibliothèque d'Érasme existent plusieurs contributions majeures :

- un article fondamental de Fritz Husner, paru en 1936 : "Die Bibliothek des Erasmus", Gedenkschrift zum 400 Todestage des Erasmus von Rotterdam. S'y trouve publié ce qu'on appelle la «Versandliste » (liste d'envoi), inventaire de la bibliothèque d'Érasme réalisé au moment de sa mort. Cet inventaire est conservé grâce au ms CVI a 71 fol. 38-45 B.U. Bâle.

- une mise au point établie par Alexandre Vanautgaerden au cours d'un colloque qui s'est tenu à Bruxelles en 1999 sous le titre "Un deuxième inventaire de la bibliothèque d'Érasme»; l'auteur y publie et analyse notamment une seconde liste manuscrite, également conservée à la BU de Bâle, qui se présente comme un complément à la «Versandliste » et comporte tous les ouvrages dus à la plume d'Érasme, les « erasmiana », présents dans sa propre bibliothèque.

- Alexandre Vanautgaerden, «Ex bibliotheca Erasmi : Catalogue des trentetrois ouvrages conservés de la bibliothèque d'Érasme et des onze ex dono connus à ce jour ", Le bibliotheche private come paradigma bibliografico, Atti del convegno internazionale, Roma, Tempio di Adriano, 10-12/10/2007, ed. Fiametta Sabba, Rome, « Il bibliotecario », 20, 2008, p. 313-362. 
avait acquis la collection d'Érasme de son vivant et lui en avait laissé l'usufruit. Cet inventaire recense quatre cent treize titres, mentionnés sans grande précision, faisant apparaître l'auteur, parfois le titre, accompagnés de quelques rares mentions d'imprimeur ou de langue. Quels sont, en regard du modèle bibliographique que nous livrent les Adages, les auteurs classiques présents dans cette bibliothèque ${ }^{15}$ ?

Parmi les Grecs se distinguent d'abord les poètes: Homère est représenté par trois éditions en grec, dont une avec commentaire (Husner, 1936, 109, 110, 114) ; Pindare par deux éditions, dont une également commentée (Husner, 1936, 105, 107) ; Théocrite par quatre éditions, dont deux en grec et l'une bilingue (Husner, 1936, 98, 99, 106, 294). Viennent ensuite les auteurs dramatiques, Aristophane dans une édition grecque (Husner, 1936, 116), Euripide dans deux éditions, également en grec (Husner, 1936, 100, 103) ; Sophocle, joint à Euripide, dans une autre édition grecque (Husner, 1936, 102). La bibliothèque d'Érasme fait aussi une large place aux philosophes et aux moralistes : Aristote y est présent en six éditions différentes, dont une grecque de Johann Bebel (Husner, 1936, p. 53, 210, 211, 212, 214, 215) ; Platon bénéficie de trois éditions, dont l'édition aldine grecque et la traduction de Marsile Ficin (Husner, 1936, 216, 217, 218) ; sept éditions de Plutarque (Husner, 1936, 57, 200, 208, 265, 266, 267, 303) et quatre de Lucien, dont l'aldine et les traductions d'Érasme (Husner, 1936, 26, 53, 118, 123), viennent compléter les œuvres des deux grands philosophes grecs. Parmi les historiens, Xénophon, dans deux éditions grecques (Husner, 1936, 204, 273), voisine avec

15 Les références aux ouvrages présents dans la bibliothèque d'Érasme seront données par rapport à la liste publiée par Husner. 
Hérodote, également dans deux éditions, dont l'une offre la traduction de Valla (Husner, 1936, 272, 311). Trois éditions de Thucydide, qui profite également d'une traduction de Valla (Husner, 1936, 276, 294, 406), côtoient l'édition grecque aldine des œuvres de Pausanias (Husner, 1936, 273). Seul Flavius Josèphe, pourtant présent sous forme de deux éditions (Husner, 1936, 193, 21), ne figure pas dans la liste des historiens des Adages. S'agissant des orateurs, Érasme possède une édition d'Isocrate (Husner, 1936, 124), deux de Démosthène, dont une en grec (Husner, 1936, 130, 131), auxquelles s'ajoutent deux éditions, dont l'aldine, de rhéteurs grecs (Husner, 1936, 127, 132). Viennent s'y joindre des autorités scientifiques, Ptolémée - trois éditions, dont une en grec (Husner, 1936, p. 101, 134, 136) - et Strabon - trois éditions également (Husner, 1939 274, 275, 303) — pour la géographie ; Galien — trois éditions, dont une grecque (Husner, 1936, 137, 138, 399) - et une édition d'Hippocrate (Husner, 1936, 90) pour la médecine. Là encore, seul Dioscoride, dont trois éditions figurent dans la bibliothèque d'Érasme (Husner, 1936, 201, 202, 203), ne fait pas partie du panthéon des auteurs des Adages. Enfin, Érasme possède également l'édition aldine grecque d'Athénée, l’un des compilateurs alexandrins dont proviennent nombre de collections de proverbes (Husner, 1936, 239).

Du côté des auteurs latins, les poètes sont en nombre : si Ovide se présente sous forme de trois éditions (Husner, 1936, 84, 85, 86), Horace (Husner, 1936, 88) et Virgile (Husner, 1936, 82) ne bénéficient que d'une seule édition, tout comme Lucain (Husner, 1936, 93) et Martial (Husner, 1936, 113). En revanche, parmi les auteurs dramatiques, seul figure Térence, avec deux éditions (Husner, 1936, 83, 117). Les historiens classiques sont massivement présents: Suétone en quatre éditions (Husner, 
1936, p. 51, 261, 262, 263), Tite-Live en trois éditions (Husner, 1936, 259, 260, 411), Tacite en deux (Husner, 1936, 269, 270) et Salluste en une seule (Husner, 1936, 308). Ils côtoient les orateurs ou épistoliers comme Cicéron - six éditions (Husner, 1936, 111, 120, 121, 122, 246, 253) -, Sénèque - trois éditions (Husner, 1936, 80, 81, 148) -, Quintilien - deux éditions, dont l'aldine (Husner, 1936, 119, 157) - et Pline le Jeune pour une seule édition (Husner, 1936, 67). S'y ajoutent des auteurs de miscellanées comme Pline l'Ancien - quatre éditions (Husner, 1936, 205, 206, 207, 412) -, AuluGelle (Husner, 1936, 281) et Macrobe (Husner, 1936, 282) ; enfin Érasme a acquis une édition de Res rusticae qui réunit Columelle, Varon et Caton (Husner, 1936, 324). Cependant, les écrivains moins classiques que sont Ausone (Husner, 1936, 156) et Apulée (Husner, 1936, p. 197), pourtant appréciés par Érasme, ne fournissent pas de moisson aux Adages.

Le recouvrement entre la bibliothèque privée d'Érasme et le catalogue des auteurs des Adages est important, la constitution de ce dernier dépendant grandement des ouvrages présents dans la bibliothèque érasmienne. Les Adages sont avant tout un ouvrage de références littéraires de première main, trouvées dans des bibliothèques, et non une compilation à partir de collections déjà existantes : mis à part Athénée, qui est effectivement présent dans la bibliothèque d'Érasme, ce n'est pas aux compilateurs que l'humaniste fait appel, mais bien à ses auteurs de prédilection. Souvent, le nombre élevé de citations d'un auteur dans les Adages correspond au nombre non négligeable d'éditions de ce même auteur dans la bibliothèque érasmienne: c'est le cas par exemple pour Homère, Aristote et Platon, ou Cicéron. 
Mais ce mouvement dialectique entre bibliographie et bibliothèque d'Érasme est relayé par d'autres courants. Certes, les Adages fonctionnent comme une sorte de catalogue de la bibliothèque privée d'Érasme, dont on peut retrouver le contenu à l'état latent dans l'ouvrage. Cependant, les Adages font également référence à ce que l'on pourrait appeler une «bibliothèque collective», paradigme de bibliothèque idéale inscrite dans le mouvement général de circulation des textes qui touche l'ensemble de l'Europe et ouvre les frontières de la République des lettres. Dans l'édition vénitienne des Chiliades, Érasme rappelle qu'il a eu recours aux manuscrits appartenant à des érudits, comme Musurus ou Aléandre, qui fréquentent le cercle de l'Académie aldine. Le texte des Adages offre un catalogue de manuscrits encore inédits, appelés à former le socle de la future bibliothèque idéale des Belles Lettres, dont les éditions aldines, décrites, dans l'essai Festina lente (Adage 1001), en une magnifique mise en abyme, offriront le reflet parfait (A.S.D., 1969, II, 3, p. 9-28). Enfin, dans la préface à l'édition parue chez Froben en 1515 (Allen, I, 269), Érasme renvoie à une autre bibliothèque, celle de William Warham. Cette épître liminaire est construite, là aussi, sur un jeu rhétorique : Érasme substitue au catalogue de la bibliothèque de Warham celui des vertus de l'archevêque anglais (decorum catalogus); la bibliothèque échappe au réel, sa richesse devenant métaphore des ressources morales de son possesseur. Érasme inscrit ainsi son œuvre dans un espace européen, qui rassemble autant l'horizon anglais, où il découvre les joies de l'érudition, que le paysage italien, où il perfectionne ses connaissances classiques.

Revenons une ultime fois sur la préface de la première édition des Adages qui serait, pour reprendre les termes de 
Marc Fumaroli, un véritable "petit traité d'art oratoire" (p. 95); elle présente la littérature, nécessité vitale (maxime quod sine litterarum commercio non video quid hec habeat vita suave), comme une mosaïque de références, "tout discours comme un carrefour visible de discours antérieurs » qui se réclame de ses sources. La bibliographie serait finalement perçue comme consubstantielle à la littérature, les auteurs cités ne conférant pas une autorité au texte qui se construit, qu'il soit celui des Adages ou quelque autre, mais lui donnant en quelque sorte son horizon culturel, le déployant dans son historicité. Au terme de ce parcours de lecture bibliographique appliqué à un grand texte humaniste, nous pourrions risquer l'hypothèse que bibliothèque et bibliographie forment les espaces nécessaires à la création de nouveaux textes, qui existent indépendamment de l'accumulation des livres à l'intérieur de la bibliothèque, indépendamment des canons établis, mais pourtant par la grâce de leur existence. Et c'est sans doute sur cette conception et cette acception érasmienne de ce que Conrad Gesner appellera bibliotheca - autant bibliographie que bibliothèque - , que nous pourrions conclure.

\section{Bibliographie}

AlLEN, P. S. 1906-1958, Opus epistolarum Desiderii Erasmi Roterodami, ed. P. S. Allen, H. M. Allen, H. W. Garrod, Oxford, 12 vol.

ARNolD, T. J. 1897, Bibliotheca erasmiana, bibliographie des cuvres d'Erasme : Adagia, Gand.

A.S.D. 1969-, Adages, Opera omnia Desiderii Erasmi Roterodami, Amsterdam, II, 1-6. 
BALAVoINE, Claudie. 1984, «Les principes de la parémiographie érasmienne », dans François Suard et Claude Buridant (dir.), Richesse du proverbe, vol. II, Lille, Université de Lille, p. 9-23.

ChOmarat, Jacques. 1980, Grammaire et rhétorique chez Érasme, Paris, Les Belles Letrres.

Fumaroli, Marc. 1980, L'âge de l'éloquence, Genève, Droz.

GeSNER, Conrad. 1545, Bibliotheca universalis sive Catalogus omnium scriptorum locupletissimus, in tribus linguis, latina, graeca et hebraica, extantium et non extantium, veterum et recentiorum in hunc usque diem, doctorum et indoctorum, publicatorum et in bibliothecis latentium... Tiguri : apud C. Froschoverum.

HUSNER, Fritz. 1936, "Die Bibliothek des Erasmus », dans Gedenkschrift zum 400 Todestage des Erasmus von Rotterdam, Bâle, p. 228-259.

Martin, Henri-Jean. 2000, La naissance du livre moderne, Paris, Éditions du Cercle de la librairie.

PhILLIPS, Margaret Mann. 1964, The Adages of Erasmus, New York, Garland.

VANAUTGAERDEN, Alexandre. 2008, «Ex bibliotheca Erasmi : Catalogue des trente-trois ouvrages conservés de la bibliothèque d'Érasme et des onze ex dono connus à ce jour », Le bibliotheche private come paradigma bibliografico, Atti del convegno internazionale, ed. Fiametta Sabba, Rome, «Il bibliotecario », 20, p. 313-362.

—. 2002, Un deuxième inventaire de la bibliothèque d'Érasme, Louvain / Paris, Peeters / Université libre de Bruxelles. 


\title{
Résumé
}

Depuis leur première publication, en 1500, jusque leurs reprises, augmentations et adaptations, les Adages d'Érasme se présentent comme l'idéale bibliographie de lectures d'autorité et de traditions. Ces autorités et la manière dont elles sont mises en lumière par Érasme et ses imprimeurs constituent en effet une sorte de liste de lectures qu'il nous faut maintenant examiner. Érasme s'assigne en effet la tâche, même si elle n'est présentée qu'en creux, d'établir un véritable catalogue (catalogus ou recensio) des auteurs d'adages, anciens et modernes. Peut-on alors parler de la bibliographie comme la représentation textuelle d'une histoire littéraire qui se donne à lire?

\begin{abstract}
As of their first publication in 1500, up until their numerous reeditions, reimpressions, revisions, and augmentations, the Adages collected by Erasmus can be read as an ideal bibliography of authoritative sentences, anecdotes, and references. The reader is allowed to consider the Erasmian collection as a reading list for authorities but also for the art of publishing authorities. For Erasmus implicitly chooses to establish a catalogue (catalogus or recensio) of all ancient and modern authors of adages. Can we, then, consider bibliography as a performance, within the text, of literary history?
\end{abstract}

